(c) 2010 IEEE. Personal use of this material is permitted. Permission from IEEE must be obtained for all other uses, in any current or future media, including reprinting/republishing this material for advertising or promotional purposes, creating new collective works, for resale or redistribution to servers or lists, or reuse of any copyrighted component of this work in other works. 


\title{
A Framework for Low Complexity Least-Squares Localization With High Accuracy
}

\author{
Junlin Yan, Christian C. J. M. Tiberius, Peter J. G. Teunissen, Giovanni Bellusci, and Gerard J. M. Janssen
}

\begin{abstract}
In this paper, a new framework is proposed for least-squares localization based on estimated ranges, covering time-difference-of-arrival (TDoA), time-of-arrival (ToA), and received signal strength (RSS) cases. The multidimensional nonlinear localization problem is first transformed to a lower dimension and then solved iteratively. Within the proposed transformed least-squares (TLS) framework, we introduce a method in which the localization problem is transformed to one dimension (1-D). In this way, compared to the classical nonlinear least-squares (NLS) type of methods, the amount of computations in each iteration is greatly reduced; a reduction of $67 \%$ for a 3-D positioning system is shown. Hence, the introduced 1-D iterative (1DI) method is fairly light on the computational load. The way to choose the 1-D parameter is proposed, and theoretical expressions for the convergence rate and the root- mean-squared error (RMSE) of the 1DI estimator are derived. Validation is performed mainly based on actual ultra-wideband (UWB) radio measurements, collected in typical office environments, with signal bandwidths varying from 0.5 to $7.5 \mathrm{GHz}$. Supplementary simulations are also included for validation. Results show that, in terms of RMSE, the 1DI method performs better than the linear least-squares (LLS) method, where the solution is obtained noniteratively, and performs similarly as NLS, especially in TDoA cases.
\end{abstract}

Index Terms-Least-squares localization, low complexity, ultrawideband (UWB).

\section{INTRODUCTION}

I $\mathrm{N}$ recent years, position information has attracted increasing attention with the success of GPS satellite navigation. Besides the value in transport and military events, positioning services find their applications in a variety of areas, such as industry, commerce, science, sports, and personal everyday life. These applications are being developed at an astonishing rate.

Manuscript received August 04, 2009; accepted May 02, 2010. Date of publication May 17, 2010; date of current version August 11, 2010. The associate editor coordinating the review of this manuscript and approving it for publication was Dr. Zhi Tian. This work was supported by the Technology Foundation STW, Applied Science Division of NWO and by the Technology Programme of the Ministry of Economic Affairs.

J. Yan and C. C. J. M. Tiberius are with the Faculty of Aerospace Engineering, Delft University of Technology, 2629 HS, Delft, The Netherlands (e-mail: J.Yan@tudelft.nl; C.C.J.M.Tiberius@tudelft.nl).

P. J. G. Teunissen is with the Faculty of Aerospace Engineering, Delft University of Technology, 2629 HS, Delft, The Netherlands. He is also with the GNSS team, Department of Spatial Sciences, Curtin University of Technology, WA 6845, Perth, Australia(e-mail: P.J.G.Teunissen@tudelft.nl).

G. Bellusci and G. J. M. Janssen are with the Faculty of EEMCS, Delft University of Technology, 2628 CD, Delft, The Netherlands (e-mail: G.Bellusci@ tudelft.nl; G.J.M.Janssen@tudelft.nl).

Digital Object Identifier 10.1109/TSP.2010.2050313
The majority of positioning systems estimate the unknown position based on (pseudo)range measurements, which can be obtained by extracting the information contained in the received signal, such as time-difference-of-arrival/time-of-arrival (TDoA/ToA) and received signal strength (RSS). In TDoA systems, a common offset $b_{c}$ in all the estimated pesudoranges, caused by the clock misalignment between the user receiver clock and the synchronized transmitter clocks, needs to be estimated, in addition to the unknown user position. For fully synchronized (ToA) or nontiming based systems (like RSS), no offset exists.

The unknown position coordinates are nonlinearly involved in the range equations and the estimation is usually done using iterative descent (ID) algorithms [1], which solve the nonlinear least-squares (NLS) problem iteratively by means of linear inference.

The NLS estimator and the maximum likelihood estimator (MLE) are equivalent, when range errors are Gaussian distributed and proper weight matrices are used. However, the NLS estimator is obtained with quite demanding computations in each iteration and the total computational load is especially heavy when a large number of iterations is required for the ID algorithms to converge. This happens when the range errors and/or the normal curvatures of the manifold, formed by the range equations, are large [1].

The localization problem can also be formulated using squared-range (SR) equations, where the unknown $x$ is involved in a linear term and a quadratic term $\lambda=x^{T} L x$, with $L$ a known matrix. Direct noniterative solutions can be obtained by means of least-squares [2]-[10] or simply by averaging [11]. In [2] and [3], the constraint $\lambda=x^{T} L x$ is partially exploited while in [4]-[10], $\lambda$ is treated as an additional unknown, independent on $x$. The final estimate is either obtained by directly using the SRs, or by using the squared-range differences (SRD), where $\lambda$, common for all SRs, is canceled. The direct least-squares (DLS) estimators are simple to compute, but none of them is the strict least-squares solution to the SR equations. Improvements on the accuracy of the DLS estimator can be found in [12] (for TDoA) and [13] (for ToA and RSS), where a Lagrange Multiplier is used in the objective function to fully account for the constraint $\lambda=x^{T} L x$. The final solution is obtained iteratively by searching the roots of a high order polynomial. Although the estimator is obtained with only 1-dimensional (1-D) iterations in root searching, the required eigenvalue decomposition in the earlier stage is also a nonlinear problem, which needs to be solved iteratively. Another low-complexity iterative solution can be found in [14], 
where the authors use only one range measurement in each iteration to update the user position.

In this paper, a novel 2-step transformed least-squares (TLS) framework is proposed, for TDoA, ToA and RSS cases. The idea is to keep the required computations low by transforming the positioning problem to lower dimensions in Step 1 and solve the remaining parameters iteratively in Step 2 . Within the TLS framework, we introduce a one dimensional iterative (1DI) method where the linear least-squares (LLS) method [4], [5] is used to transform the problem to one dimension (1-D) in Step 1 and the Gauss-Newton (GN) method is used to estimate the 1-D unknown in Step 2. The issue of parameter choice in Step 1 is discussed; the theoretical expression for the convergence rate and the root mean squared error (RMSE) for the 1DI estimator are derived; the numbers of required computations for the 1DI solution and the NLS solution in each iteration are calculated. Compared to the NLS estimator obtained using the GN method, the 1DI method saves up to $67 \%$ of the computations in each iteration. Validation is performed mainly based on actual ultrawideband (UWB) range measurements, together with some supplementary simulations. Results show that the RMSE of the 1DI estimator is smaller than that of the DLS estimator, and is close to the RMSE of the NLS estimator, especially in TDoA cases. Please note that our work differs from [14] in the sense that, in each iteration, we reduce the number of unknowns, while [14] reduces the number of measurements involved.

The paper is organized as follows. Section II introduces the positioning models. In Section III, the details about the TLS framework are given. The validation setup is described in Section IV, and in Section V the obtained results are shown. Finally, in Section VI, concluding remarks are given.

\section{POSITIONING MODELS}

From now on, we will use the term TDoA for the cases where the user receiver clock is asynchronous to the transmitter clocks and the term ToA/RSS for the cases where all the clocks are synchronized or no timing information is required for ranging. Note that, in TDoA cases, with the additional unknown $b_{c}$, one extra measurement is needed for estimation, as compared to ToA/RSS cases.

\section{A. Original Model}

With unsquared range measurements, the (general) model can be written as

$$
\underline{y}=A(x)+\underline{e}
$$

where $(\cdot)$ indicates a random variable, $y \in R^{m}$ is the measurement vector, $x$ is the unknown vector, $\overline{A(\cdot)}$ is a nonlinear mapping, and $\underline{e} \in R^{m}$ is the measurement error vector. In TDoA, $x=\left[x_{u}^{T}, b_{c}\right]^{T}$ where $x_{u} \in R^{n}$ is the unknown position, $b_{c}$ is the offset. In ToA/RSS, $x=x_{u}$ and $b_{c}=0$ (left out).

For one single measurement $\underline{y}_{i}$, containing the error $\underline{e}_{i}$, the equation reads

$$
\underline{y}_{i}=\underbrace{\left\|x_{u}-x_{i}\right\|}_{d_{i}}+b_{c}+\underline{e}_{i}, \quad i=1 \ldots m
$$

where $x_{i}$ is the position vector of $i$ th transmitter, $d_{i}=\left\|x_{u}-x_{i}\right\|$ is the true distance between the $i$ th transmitter-receiver pair, and $\|\cdot\|=\sqrt{(\cdot)^{T}(\cdot)}$.

Estimating $\hat{x}_{u}$ and $\hat{b}_{c}$ from a measurement set $y$, can be done based on the NLS theory by searching the minimum of the objective function

$$
\underline{\hat{x}}=\arg \min _{x} F(x)=\arg \min _{x}\|\underline{y}-A(x)\|_{W}^{2}
$$

where $\|\cdot\|_{W}=\sqrt{(\cdot)^{T} W(\cdot)}$ denotes a weighted norm, with the weight matrix $W$. Generally, $W$ is chosen as the inverse of the measurement variance matrix, i.e., $W=Q_{y y}^{-1}=Q_{e e}^{-1}$. Due to the nonlinear nature of the model (2), solution (3) is usually obtained by the ID methods.

\section{B. Squared-Range Difference Model}

The SRD model reviewed in this section is a linear model, based on which the unknown vector $x$ can be estimated using standard LLS theory. The SRD model is obtained as follows.

First, a squaring operation is taken on both sides of (2) to get rid of the square root, for $i=1, \ldots, m$

$$
\underline{y}_{i}^{2}=x_{u}^{T} x_{u}-b_{c}^{2}-2 x_{i}^{T} x_{u}+2 \underline{y}_{i} b_{c}+x_{i}^{T} x_{i}+2 d_{i} \underline{e}_{i}+\underline{e}_{i}^{2} .
$$

The term $x_{u}^{T} x_{u}-b_{c}^{2}$, common in all $m$ SRs, can be canceled by taking differences between SRs. Without loss of generality, it is assumed that the $m$ th SR is picked as the reference. Subtracting the reference SR from all other $m-1$ SRs, it holds after arranging

$$
\begin{aligned}
\underline{y}_{i}^{2}-\underline{y}_{r}^{2}-x_{i}^{T} & x_{i}+x_{r}^{T} x_{r}=-2\left(x_{i}-x_{r}\right)^{T} x_{u} \\
& +2\left(\underline{y}_{i}-\underline{y}_{r}\right) b_{c}+2 d_{i} \underline{e}_{i}-2 d_{r} \underline{e}_{r}+\underline{e}_{i}^{2}-\underline{e}_{r}^{2}
\end{aligned}
$$

where $i \neq r$ and $r=m$. Considering all $m-1$ SRD measurements, the matrix form of (5) is

$$
\underline{y}_{L}=\underline{A}_{L} x+\underline{e}_{L}
$$

where $\underline{y}_{L}$ is an $(m-1) \times 1$ vector with $\underline{y}_{L, i}=\underline{y}_{i}^{2}-\underline{y}_{r}^{2}-$ $x_{i}^{T} x_{i}+x_{r}^{T} x_{r}$, and $\underline{e}_{L}$ is an $(m-1) \times 1$ vector with $\underline{e}_{L, i}=$ $2 d_{i} \underline{e}_{i}-2 d_{r} \underline{e}_{r}+\underline{e}_{i}^{2}-\underline{e}_{r}^{2}$. The expression for the matrix $\underline{A}_{L}$ is

$$
\begin{aligned}
& \underline{A}_{L} \stackrel{\text { TDoA }}{=}-2\left[\begin{array}{cc}
\left(x_{1}-x_{r}\right)^{T} & \underline{y}_{r}-\underline{y}_{1} \\
\vdots & \vdots \\
\left(x_{m-1}-x_{r}\right)^{T} & \underline{y}_{r}-\underline{y}_{m-1}
\end{array}\right] \\
& A_{L} \stackrel{\mathrm{ToA} / \mathrm{RSS}}{=}-2\left[\begin{array}{c}
\left(x_{1}-x_{r}\right)^{T} \\
\vdots \\
\left(x_{m-1}-x_{r}\right)^{T}
\end{array}\right] \text {. }
\end{aligned}
$$

Please note that in ToA/RSS, $A_{L}$ does not contain any random variables.

In the end, since (6) is linear in $x_{u}$ and $b_{c}$, the estimation can be done directly without any iterations using the LLS method [4] (for TDoA) [5] (for ToA/RSS), and the estimator can be given as $\underline{\hat{x}}=\left(\underline{A}_{L}^{T} W_{L} \underline{A}_{L}\right)^{-1} \underline{A}_{L}^{T} W_{L} \underline{y}_{L}$.

The nonlinear squaring operation generally makes the statistics of $\underline{e}_{L}$ different from $\underline{e}$. This, however, does not introduce too much trouble for (rigorous) weighted least-squares estimation, where only the first two moments of $\underline{e}_{L}$ are required, 
rather than the full distribution, to calculate the weight matrix $W_{L}=Q_{e_{L} e_{L}}^{-1}$. The variance matrix $Q_{e_{L} e_{L}}$ can be derived based on the statistics of $\underline{e}$, e.g., in [10] under the assumption $\underline{e} \sim N\left(0, Q_{e e}\right)$. Moreover, the presence of $d_{i}$ in $\underline{e}_{L, i}$ propagates into the expression of $W_{L}=Q_{e_{L} e_{L}}^{-1}$. Due to the fact that the information on $d_{i}$ is not available in practice, one has to replace it with $y_{i}$ to evaluate the weight matrix. As long as $W_{L}=Q_{e_{L} e_{L}}^{-1}$ is chosen, the choice of the reference in (5) does not affect the final results, which is partially verified in [10].

\section{TLS FRAMEWORK}

In this section, the new TLS framework is introduced. Although the default dimension of the user position is $n=3$, the following derivations are valid for general cases with $n$ dimensions.

\section{A. Basic Idea}

The idea consists of two steps. In Step 1, the key point is to reformulate or re-parametrize the positioning problem to reduce the number of unknown parameters. It is assumed that one can transform the unknown vector $x$ to another unknown vector $z$ of less dimensions, i.e., $x=L(z)$. We will come back to the point of how to obtain $x=L(z)$, shortly. A new model is then formed based on (1)

$$
\underline{y}=A(x)+\underline{e}=A(L(z))+\underline{e} .
$$

In Step 2, the least-squares solution to (9) is obtained as

$$
\underline{\hat{x}}=L(\underline{\hat{z}})=L\left(\arg \min _{z}\|\underline{y}-A(L(z))\|_{Q_{y y}^{-1}}^{2}\right)
$$

which can be done using the ID methods.

If the mapping $x=L(z)$ is error-free, the estimator (10) is better than (3), in both the senses of accuracy and computational complexity, since the objective function in (10) contains less unknowns than (3) due to the additional information $x=L(z)$. Note that the computational complexity is reduced the most when $z$ is a scalar (1-D vector).

Now the unsolved issue is how to find the mapping $L(z)$ in Step 1 . In practice, an error-free mapping is usually not available and one has to estimate it using the data at hand. Hence, the mapping in Step 1 becomes $L(z, y)$. Since $y$ contains measurement error $\underline{e}$ in (1), the mapping can also be written as $L(z, \underline{e})$, and (10) becomes

$$
\underline{\hat{x}}=L(\underline{\hat{z}}, \underline{e})=L\left(\arg \min _{z}\|\underline{y}-A(L(z, \underline{e}))\|_{Q_{y y}^{-1}}^{2}, \underline{e}\right) .
$$

The estimation of $L(z, \underline{e})$ depends on the formulated nonlinear problem to solve. Fortunately, for positioning problems, $L(z, \underline{e})$ can be obtained, e.g., based on direct methods. This point will be clearer with the aid of an example in the next section, where, as a realization of the TLS framework, the 1DI method is introduced. In the 1DI method, $z$ is a scalar, $L(z, \underline{e})$ is estimated based on the LLS method, and the objective function in (11) is minimized using the GN method. We should note that the TLS framework provides a general way to solve the positioning problem, and the ways to obtain $L(z, \underline{e})$ and the final solution are not limited to the LLS method and the GN method, respectively.

\section{B. An Example: 1DI Method}

Actually there exists an unlimited number of choices for $z$ in $L(z, \underline{e})$, and how to choose the parameter is discussed in Section III-C. In the following derivations, it is assumed that for TDoA, the chosen parameter is $z=b_{c}$ while for ToA/RSS, the chosen parameter is one of the unknowns, e.g., $z=x_{k}$, the $k$ th element of $x$.

1) $T D o A$ : Based on (6), it follows

$$
\begin{aligned}
\underline{\hat{x}}_{u}\left(b_{c}\right) & =\arg \min _{x_{u}}\left\|\underline{y} L-B x_{u}-\underline{\delta y}_{r} b_{c}\right\|_{W_{L}}^{2} \\
& =E \underline{y}_{L}+E \underline{\delta}_{r} b_{c}
\end{aligned}
$$

where $B$ is an $(m-1) \times n$ matrix that contains all columns of $\underline{A}_{L}$, except the last column $-\underline{\delta y}$, with $\underline{\delta y}_{r}=-2\left[\underline{y}_{1}-\right.$ $\left.\underline{y}_{r}, \ldots, \underline{y}_{m-1}-\underline{y}_{r}\right]^{T}$, and $E=\left(\bar{B}^{T} W_{L} B\right)^{-\frac{{ }_{1}^{1}}{B}} B^{T} W_{L}$. The key term $L\left(b_{c}, \underline{e}\right)$ in Step 1 can then be written as

$$
L\left(b_{c}, \underline{e}\right)=\left[\underline{\hat{x}}_{u}\left(b_{c}\right)^{T}, b_{c}\right]^{T} .
$$

Further denote, for $i=1, \ldots, m$,

$$
P_{i}\left(b_{c}, \underline{e}\right)=A_{i}\left(L\left(b_{c}, \underline{e}\right)\right)=\left\|E \underline{y}_{L}+E \underline{\delta}_{r} b_{c}-x_{i}\right\|+b_{c}
$$

then, based on (2), a new model can be given as

$$
\underline{y}=P\left(b_{c}, \underline{e}\right)+\underline{e}_{P}
$$

where $\underline{e}_{P}=A(x)-P\left(b_{c}, \underline{e}\right)+\underline{e}$. The effect of $\underline{e}_{P}$ on the final estimator is addressed in Section III-D.

In Step 2, the parameter $b_{c}$ can be estimated based on (11) as

$$
\underline{\hat{b}}_{c}=\arg \min _{b_{c}}\left\|\underline{y}-P\left(b_{c}, \underline{e}\right)\right\|_{W}^{2}
$$

where $W=Q_{y y}^{-1}$, see (11).

Equation (16) is a nonlinear problem, which can be solved by means of ID algorithms, e.g., the Steepest Descent method, the Newton method, the GN method and the Trust Region method. Concerning estimation quality, these methods provide equivalent solutions when they successfully converge. In the 1DI method, the GN method is chosen to solve (16), since it is widely used in positioning systems, e.g., in GPS [15]. Furthermore, the GN method does not have the zigzag problem of the Steepest Descent method, and it is of lower complexity without requiring the evaluation of second order derivatives of $A(x)$, as compared to the Newton method.

First, (15) is linearized as

$$
\underline{y} \approx P\left(b_{c}^{0}, \underline{e}\right)+\partial_{b_{c}} P\left(b_{c}^{0}, \underline{e}\right)\left(b_{c}-b_{c}^{0}\right)+\underline{e}_{P}
$$

where $b_{c}^{0}$ is the initial guess for $b_{c}$, which can be obtained using a direct method. 
Based on (17), the parameter $b_{c}$ can be estimated iteratively and in the $(j+1)$ th iteration, the estimate is updated based on the approximation of (16)

$\underline{\hat{b}}_{c}^{j+1}=\arg \min _{b_{c}}\left\|\underline{y}-P\left(b_{c}^{j}, \underline{e}\right)-\partial_{b_{c}} P\left(b_{c}^{j}, \underline{e}\right)\left(b_{c}-b_{c}^{j}\right)\right\|_{W}^{2}$.

The mechanics can be summarized as follows.

Step 1) Estimate $L\left(b_{c}, \underline{e}\right)$;

1a. choose $b_{c}$ as the parameter to refine;

1b. calculate $E, \underline{\delta y}$ in (12);

1c. define the new model $\underline{y}=P\left(b_{c}, \underline{e}\right)+\underline{e}_{P}$;

Step 2) Calculate $x$ based on the refinement of $b_{c}$;

2a. choose an initial guess $b_{c}^{0}$;

2b. calculate $\partial_{b_{c}} P\left(b_{c}^{0}, e\right)$ based on (14);

2c. calculate

$$
\hat{b}_{c}=\frac{\partial_{b_{c}} P^{T}\left(b_{c}^{0}, e\right) W\left(y-P\left(b_{c}^{0}, e\right)\right)}{\partial_{b_{c}} P^{T}\left(b_{c}^{0}, e\right) W \partial_{b_{c}} P\left(b_{c}^{0}, e\right)}+b_{c}^{0}
$$

and terminate the iteration if

$$
\left\|\hat{b}_{c}-b_{c}^{0}\right\|^{2}<\epsilon
$$

otherwise, choose $b_{c}^{0}=\hat{b}_{c}$, and go to Step $2 \mathrm{~b}$.

2d. finally, calculate $\hat{x}_{u}$ based on (13).

Note that the norm in (20) should be taken w.r.t. the inverse of the variance of $\hat{b}_{c}$ to make the it insensitive to scale changes [1]. However, in this paper, we keep the norm unweighted for simplicity.

2) $T o A / R S S$ : Here, the offset $b_{c}$ does not exist, which makes it a bit simpler than TDoA. The derivations for TDoA can be easily adopted for ToA/RSS and only minor changes are required.

For ToA/RSS, it is assumed that the $k$ th element of $x$ is chosen, the other elements of $x$ can be estimated as functions of $x_{k}$ as

$$
\begin{array}{r}
{\left[\underline{\hat{x}}_{1}\left(x_{k}\right), \ldots, \underline{\hat{x}}_{k-1}\left(x_{k}\right), \underline{\hat{x}}_{k+1}\left(x_{k}\right), \ldots, \underline{\hat{x}}_{n+1}\left(x_{k}\right)\right]^{T}} \\
=\left(B^{T} W_{L} B\right)^{-1} B^{T} W_{L}\left(\underline{y}_{L}+C x_{k}\right)
\end{array}
$$

where $B$ is an $(m-1) \times(n-1)$ matrix that contains all columns of $\underline{A}_{L}$, except the $k$ th column $-C$.

Further denote $D_{(n-1) \times(m-1)}=\left(B^{T} W_{L} B\right)^{-1} B^{T} W_{L}$, $E_{n \times(m-1)}=\left[D_{1}^{T}, \ldots, D_{k-1}^{T}, 0, D_{k+1}^{T}, \ldots, D_{n-1}^{T}\right]^{T}$, and $F_{n \times 1}=[0, \ldots, 0, \underbrace{1}_{k-\text { th }}, 0 \ldots, 0]^{T}$, with $D_{1}$ stands for the first row of $D$. Note that $E$ here is different from the one in (12). The key expressions can be given as

$$
\begin{aligned}
L\left(x_{k}, \underline{e}\right) & =\underline{\hat{x}}_{u}\left(x_{k}\right)=E \underline{y}_{L}+(E C+F) x_{k}, \\
P_{i}\left(x_{k}, \underline{e}\right) & =\left\|E \underline{y}_{L}+(E C+F) x_{k}-x_{i}\right\| \\
\partial_{x_{k}} P_{i}\left(x_{k}, \underline{e}\right) & =\frac{\left(E \underline{y}_{L}+(E C+F) x_{k}-x_{i}\right)^{T}(E C+F)}{\left\|E \underline{y}_{L}+(E C+F) x_{k}-x_{i}\right\|},
\end{aligned}
$$

$$
\hat{x}_{k}=\frac{\partial_{x_{k}} P^{T}\left(x_{k}^{0}, e\right) W\left(y-P\left(x_{k}^{0}, e\right)\right)}{\partial_{x_{k}} P^{T}\left(x_{k}^{0}, e\right) W \partial_{x_{k}} P\left(x_{k}^{0}, e\right)}+x_{k}^{0} .
$$

The mechanics of the 1DI method in the ToA/RSS case can be derived in a very similar way as for TDoA, and is therefore not repeated here.

\section{Parameter Choice}

In Step 1, $z$ can be chosen as a linear combination of the elements of $x$ or even a nonlinear combination, e.g., $x^{T} L x$. The best parameter to iterate on should be chosen based on the requirements of the application, e.g., minimum RMSE or maximum likelihood. However, finding the parameter is in general a nonlinear problem and can be computationally involved. This can jeopardize the low complexity nature of the 1DI method, which is a favorable property compared with the NLS estimator. Simplifications are therefore desirable.

Based on the fact that the major reduction of RMSE of the 1DI estimator, compared to the LLS estimator, comes from the refinement of the parameter chosen in Step 1, we propose to choose a single element in $x$, which has worst precision.

1) TDoA: In this case, the design matrix $\underline{A}_{L}$ in (8) is usually badly-conditioned, due to the last column $-2\left[\underline{y}_{r}-\underline{y}_{1}, \ldots, \underline{y}_{r}-\right.$ $\left.\underline{y}_{(m-1)}\right]$, corresponding to the unknown $b_{c}$. If the parameter is chosen as $x_{k}, k=1, \ldots, n$, the matrix $\underline{B}$ in (12) remains badconditioned, and the estimation (13) is of very bad accuracy. Thus, in TDoA cases, we propose to choose $b_{c}$ as the parameter to be refined in Step 2. The resulting design matrix $B$ is in a much better condition and is free of random variables.

2) $T o A / R S S$ : Here, the situation is different, since the design matrix $A_{L}$ is usually in a reasonable to good condition. The parameter $x_{k}$ can be chosen such that the RMSE can be minimized, based on the expressions given in Section III-D. However, to calculate the RMSE value for $n$ different parameter choices can be computationally heavy. Fortunately, in most of the indoor situations, one can simply choose the height ( $z$-coordinate) as the parameter to refine. The reason is that most of the indoor rooms have smaller heights than lengths and widths, and the transmitters are usually installed in the corners of a room or even all on the ceiling above the user (to avoid blockage). This type of system geometry makes the confidence region in the local height direction much more elongated than in the horizontal plane. This can be easily verified with the theoretical error analysis in [16], for NLSE estimators. As a result, the estimated height is usually of worst precision. This point is verified in Section V, by comparing the outcomes of two different ways of choosing $x_{k}$.

\section{Error Analysis}

In this section, the RMSE of the 1DI estimator is derived, and we assume that the elements in $\underline{e}$ are independent and $\mathrm{E}\{\underline{e}\}=0$. Assume that the unknown parameter $z$ in (9) can be related to $y$ with a nonlinear mapping $M(\cdot)$, i.e., $z=M(y)$, and we define $\underline{\hat{z}}=M(\underline{y})$. Taylorizing $M(\underline{y})$ at $y=E\{\underline{y}\}$, with $\underline{e}=\underline{y}-y$, gives

$$
\underline{\hat{z}}=M(y)+\partial_{y} M(y)^{T} \underline{e}+\frac{1}{2} \underline{e}^{T} \partial_{y y^{T}}^{2} M(y) \underline{e}+\cdots .
$$


Define the bias in $\underline{\underline{\hat{z}}}$ as $\mu_{\hat{\underline{z}}}=\underline{\hat{z}}-z$, which is given, together with the variance of $\underline{\hat{\hat{z}}}$, as

$$
\begin{aligned}
\mu_{\hat{z}} & =\mathrm{E}\left\{\underline{\mu}_{\hat{z}}\right\}=\frac{1}{2} \operatorname{trace}\left\{\partial_{y y^{T}}^{2} M(y) Q_{e e}\right\} \\
Q_{\hat{z} \hat{z}} & =Q_{\mu_{\hat{z}} \mu_{\hat{z}}}=\mathrm{E}\left\{\left(\underline{\mu}_{\hat{z}}-\mu_{\hat{z}}\right)\left(\underline{\mu}_{\hat{z}}-\mu_{\hat{z}}\right)^{T}\right. \\
& =\partial_{y} M(y)^{T} Q_{e e} \partial_{y} M(y)
\end{aligned}
$$

where the terms with order higher than 2 are ignored.

1) TDoA: In this case, $z=b_{c}$, and based on (13), one obtains $\underline{\hat{\hat{x}}}_{u}\left(\underline{\hat{b}}_{c}\right)=L\left(\underline{\hat{b}}_{c}, \underline{e}\right)=G \underline{y} \underline{y}_{L}+G \delta \underline{y} \underline{\hat{b}}_{c}+F \underline{\hat{b}}_{c}$, where $G_{(n+1) \times(m-1)}=\left[E^{T}, 0\right]^{T}, F_{(n+1) \times 1}=[0, \ldots, 0,1]^{T}$. Denoting the error term $\underline{\mu}_{\hat{x}}=\underline{\hat{x}}-x$ and $\underline{\delta e}_{r}=\underline{\delta y} \underline{y}_{r}-\delta y_{r}=$ $-2\left[\underline{e}_{1}-\underline{e}_{r}, \ldots, \underline{e}_{m-1}-\underline{e}_{r}\right]^{T}$, the bias and variance of $\underline{\hat{x}}$ can be given based on (27) and (28) as,

$$
\begin{aligned}
\mu_{\hat{x}}= & \mathrm{E}\{\underline{\hat{x}}-x\}=G \mu_{e_{L}}+G K \partial_{y} M(y) \\
& +\left(G \delta y_{r}+F\right) \frac{1}{2} \operatorname{trace}\left\{\partial_{y y^{T}}^{2} M(y) Q_{e e}\right\} \\
Q_{\hat{x} \hat{x}}= & \mathrm{E}\left\{\left(\underline{\hat{x}}-x-\mu_{\hat{x}}\right)\left(\underline{\hat{x}}-x-\mu_{\hat{x}}\right)^{T}\right\} \\
= & G \mathrm{E}\left\{\underline{e}_{L} \underline{e}_{L}^{T}\right\} G^{T}+J+J^{T}-\mu_{\hat{x}} \mu_{\hat{x}}^{T} \\
& +\left(G \delta y_{r}+F\right) \partial_{y} M(y)^{T} Q_{e e} \partial_{y} M(y)\left(G \delta y_{r}+F\right)^{T}
\end{aligned}
$$

where $J=\mathrm{E}\left\{G \underline{e}_{L} \underline{\mu}_{\hat{b}_{c}}^{T}\left(G \delta y_{r}+F\right)^{T}\right\}$, and the fact that $G \underline{e}_{L}+\left(G \delta y_{r}+F\right) \underline{\mu}_{\hat{b}_{c}} \stackrel{\underline{\mu}_{\hat{x}}}{=}$ has been used. The details of the terms $\partial_{y} M(y), \partial_{y y^{T}}^{2} M(y), K, \mathrm{E}\left\{\underline{e}_{L} \underline{e}_{L}^{T}\right\}$, and $J$ are derived in Appendix A.

Based on (29) and (30), the RMSE can be written as

$$
\begin{aligned}
\operatorname{RMSE}_{\hat{x}} & \\
= & \operatorname{trace}\left\{Q_{\hat{x} \hat{x}}\right\} \\
& +\mu_{\hat{x}}^{T} \mu_{\hat{x}} \operatorname{trace}\left\{G \mathrm{E}\left\{\underline{e}_{L} \underline{e}_{L}^{T}\right\} G^{T}+J+J^{T}\right. \\
& \left.+\left(G \delta y_{r}+F\right) \partial_{y} M(y)^{T} Q_{e e} \partial_{y} M(y)\left(G \delta y_{r}+F\right)^{T}\right\}
\end{aligned}
$$

The first term $G \mathrm{E}\left\{\underline{e}_{L} \underline{e}_{L}^{T}\right\} G^{T}$ comes from the error in the estimation of $L(z, \underline{e})$ in Step $1, J+J^{T}$ represent the cross terms of the estimation errors in Step 1 and Step 2, and the last term comes from the estimation errors in Step 2. Equation (31) requires knowledge of the true value $x$, which is not available in practice. Therefore, the estimate $\hat{x}$ can be used instead. If one wants to use the theoretical RMSE expression for parameter choice in Step 1, before $\hat{x}$ is estimated, the initial guess obtained using a direct method can be used instead.

2) $T o A / R S S$ : Here, one has $z=x_{k}$ and it can be shown in a similar way that

$$
\begin{aligned}
\mu_{\hat{x}}= & E \mu_{e_{L}}+(E C+F) \frac{1}{2} \operatorname{trace}\left\{\partial_{y y^{T}}^{2} M(y) Q_{e e}\right\} \\
Q_{\hat{x} \hat{x}}= & E E\left\{\underline{e}_{L} \underline{e}_{L}^{T}\right\} E^{T}+J+J^{T}-\mu_{\hat{x}} \mu_{\hat{x}}^{T} \\
& +(E C+F) \partial_{y} M(y)^{T} Q_{e e} \partial_{y} M(y)(E C+F)^{T}
\end{aligned}
$$

and

$$
\begin{aligned}
\operatorname{RMSE}_{\hat{x}}= & \operatorname{trace}\left\{E \mathrm{E}\left\{\underline{e}_{L} \underline{e}_{L}^{T}\right\} E^{T}+J+J^{T}\right. \\
& \left.+(E C+F) \partial_{y} M(y)^{T} Q_{e e} \partial_{y} M(y)(E C+F)^{T}\right\} .
\end{aligned}
$$

The terms $\partial_{y} M(y), \partial_{y y^{T}}^{2} M(y)$ and $J$ in ToA/RSS cases are commented on, in Appendix A.

\section{E. Convergence Rate}

Recall from (19) and (25), the chosen parameter is updated in the $j+1$ th iteration as

$$
z^{j+1}=\frac{\partial_{z} P^{T}\left(z^{j}, e\right) W\left(y-P\left(z^{j}, e\right)\right)}{\partial_{z} P^{T}\left(z^{j}, e\right) W \partial_{z} P\left(z^{j}, e\right)}+z^{j} .
$$

Taylorizing (35) at the final solution $\hat{z}$, together with $\partial_{\hat{z}} P^{T}(\hat{z}, e) W(y-P(\hat{z}, e))=0$, gives

$$
\begin{aligned}
z^{j+1}-\hat{z}=\frac{\partial_{z^{2}}^{2} P^{T}(\hat{z}, e) W(y-P(\hat{z}, e))}{\partial_{z} P^{T}(\hat{z}, e) W \partial_{z} P(\hat{z}, e)}\left(z^{j}-\hat{z}\right) & \\
& +o\left(z^{j}-\hat{z}\right) .
\end{aligned}
$$

With $z^{j}$ close to $\hat{z}$, the term $o\left(z^{j}-\hat{z}\right)$ can be ignored, and the 1DI method has a linear rate of convergence. Note that (36) is a special case of the general expression in [1].

\section{F. Flop Count}

A flop is defined as a floating point operation, e.g., an add, a multiplication or a square root. The number of required flops to calculate the 1DI estimator (11) and the NLS estimator (3) obtained using the GN method, are compared in this section, for both TDoA and ToA/RSS, based on the number of flops required in each iteration, as derived in Appendix B.

1) TDoA:

$$
\begin{aligned}
\text { flops }_{1 \mathrm{DI}}= & 2 m^{2}+3 m n+9 m+2 n+2 \\
\text { flops }_{G N}= & n^{3} / 3+2 m^{2} n+2 m n^{2}+2 n^{2} \\
& +7 m n+4 m+6 n+4 / 3
\end{aligned}
$$

2) $T o A / R S S:$

$$
\begin{aligned}
\text { flops }_{1 \mathrm{DI}}= & 2 m^{2}+3 m n+7 m+2 n+2, \\
\text { flops }_{G N}= & n^{3} / 3+2 m^{2} n+2 m n^{2}+n^{2} \\
& +6 m n+m+2 n-1
\end{aligned}
$$

Note that with a fixed $n$, if $m$ is very large, the terms $2 m^{2}$ and $2 m^{2} n$ dominate (37) -(40). The ratio flops $_{1 D I} /$ flops $_{G N}$ gets close to $1 / n$. With $n=3$ for 3 -D positioning, the largest amount of reduction is about $67 \%$.

One may argue that if the number of flops, required to obtain the initial guess, is dominating the total computational load, then the saved computations by using the 1DI method may be negligible. Fortunately, the major computations involved in this initialization stage are no more than the major computations in one iteration of the GN method with full dimension. Suppose that the initial guess is obtained with the LLS method, the number of rows in $A_{L}$ is $m-1$, and the dominant term in the flop count becomes $2(m-1)^{2} n$, smaller than the one for the GN method. Note that the estimation of $L(z, \underline{e})$ required by the 1DI method 
can also be done based on the same direct method, and no extra computations are involved.

\section{VALIDATION SETUP}

Validation work is done mainly based on actual UWB range measurements, together with some complementary simulations.

\section{A. Actual UWB Range Measurements}

The validation using actual data can be described with the following steps:

1. Collect range measurements using actual UWB radio signals, together with the corresponding true ranges (ground truth), physically measured with a laser disto tool.

The measurement campaign is carried out in the EE-MCS building of Delft University of Technology using vertically polarized, omnidirectional biconical antennas placed $1.5 \mathrm{~m}$ above the floor. The distances between the transmitter and the receiver are between 2 and $15 \mathrm{~m}$ with LOS propagation. A total of 400 range measurements, each corresponding to different transmitter and receiver positions, were collected. The system is capable of covering the bandwidth between 3.1 and $10.6 \mathrm{GHz}$ allowed by the FCC for UWB radio transmissions [17], and the details can be found in [18].

The Channel Impulse Response (CIR) $h(t)$ is estimated by deconvolving the received signal in the frequency domain using the inverse filtering technique. The spectrum of the received signal is divided by the one of the reference signal, measured at a distance of one meter, in the absence of reflections. In this way, it is possible to take into account the transmit and receive antenna transfer functions and the characteristics of the other system components. The division is done only for the frequency band of interest, the bandwidth ranging from 0.5 to $7.5 \mathrm{GHz}$; the rest of the spectrum is filled with zeros. To reduce the leakage problem when transforming the signal back to the time domain, a Hamming window is used, which provides sidelobes less than $-43 \mathrm{~dB}$.

The distance is estimated by multiplying the speed of light in air by the ToA of the first path, defined here as the first local maximum of the envelope of the estimated channel, with amplitude within $20 \mathrm{~dB}$ from the strongest peak. In our system, the receiver and the transmitter are actually synchronized, and therefore no clock offset exists. However, to mimic a TDoA system, we simply assume the presence of a clock offset (with true value $b_{c}=0$ ), and treat it as an additional unknown. The RSS measurements are not included in this paper, due to limited space.

2. Determine bias and variance of the range measurements, based on the model introduced in [18].

Under LoS condition, the range errors are usually modeled as independent Gaussian variables [19]. It has also been reported that the mean vector and variance matrix of the error $\underline{e}$ are dependent on the distance vector $d$ and the used bandwidth $B$ [18], i.e., $\underline{e} \sim N_{m}\left(\mu(B, d), Q_{e e}(B, d)\right)$, where

$$
\begin{aligned}
\mu_{i}\left(B, d_{i}\right) & =0.0148 \exp (-B / 0.48) d_{i} \quad[\mathrm{~m}] \\
\sigma_{i}\left(B, d_{i}\right) & =\sqrt{\left[Q_{e e}(B, d)\right]_{i i}} \quad[\mathrm{~m}]
\end{aligned}
$$

$$
\begin{aligned}
& =0.016\left(0.64 \exp (-B / 0.6) d_{i}^{1.5}+1\right), \\
\left.Q_{e e}(B, d)\right]_{i j} & =0, \quad i \neq j .
\end{aligned}
$$

with $d$ in meter and $B$ in GHz. In our validation, the model in [18] is used, and the bias $\mu(B, d)$ is corrected for, i.e., $\underline{e} \sim N_{m}\left(0, Q_{e e}(B, d)\right)$.

We should note that although the true ranges are collected during the measurement campaign, they are not known in practice. Therefore, to make the validation more realistic, the true distance $d_{i}$ in (41) and (43) is replaced by $\underline{d}_{i}$, which can be calculated using the initial guess in TDoA cases and can be directly chosen as range measurements in ToA/RSS cases.

3. Construct the system geometry.

As the actual UWB radio measurements are restricted to a single link (1 Tx, $1 \mathrm{Rx})$, a 3D positioning system is created virtually by choosing sets of 5 range measurements. Without loss of generality, the user is placed at the origin. For the $i$ th transmitter, $i=1, \ldots 5$, spherical coordinates $\left(d_{i}, \theta_{i}, \phi_{i}\right)$ are assigned, see Fig. 1 , where $d_{i}$ is the true distance, obtained in Step 1, $\theta_{i}$ and $\phi_{i}$ are randomly chosen from the intervals shown in Table I. Problematic geometries that make the positioning unsolvable, e.g., with all transmitters on a line, are avoided.

4. Perform positioning using the LLS method, the 1DI method, and the GN method [solving (3)], in weighted cases. The weight matrices are evaluated based on the results obtained in Step 2.

For ToA, with 1DI, two different setups of choosing the parameter to iterate are tested, as suggested in Section III.C. In Setup 1, the coordinate on the $z$ axis (the height) is chosen, while in Setup 2, the parameter is chosen out of the $k$ elements of $x$ as the one that minimizes (34), which is evaluated using the initial guess.

For ToA, the initial guess is obtained using the LLS method. For TDoA, to avoid the numerical problem with the original LLS method, we adapt it based on the idea of [2]:

a) Obtain the mapping (13).

b) Plugging $L\left(b_{c}, \underline{e}\right)$ into the smallest (pseudo)range measurement in (1), ending up with a quadratic equation in $b_{c}$.

c) Solving the equation gives us two candidates for $b_{c}$ and therefore two candidates for $x$. The initial guess is picked as: $\hat{x}=\arg \min _{x_{1}, x_{2}}\|\underline{y}-A(x)\|_{Q_{y y}^{-1}}^{2}$.

Furthermore, the threshold $\epsilon$ in (20) is chosen as $10^{-24}$, and the algorithms will be intentionally terminated if the number of spent iterations hits 50 . The same rules hold for the GN method.

With a fixed signal bandwidth, a total number of 50 000 tests are performed. The performance of the positioning algorithms is evaluated based on the RMSE of the corresponding position estimates, which quantifies how far the estimates are from the true position. The RMSE of the estimator is obtained empirically as $\operatorname{RMSE}=\sqrt{1 /(N-1) \sum_{l=1}^{N}\left\|\hat{x}_{u}^{l}-x_{u}\right\|^{2}}$, with $x_{u}$ the true position, $N=50000$, and $\hat{x}_{u}^{l}$ the estimates obtained 
TABLE I

The InTERVALS of ZeNith ANGLE $\theta$ AND AZIMUth ANGLE $\phi$

\begin{tabular}{|l||c|c|}
\hline$i$ & $\theta$ & $\phi$ \\
\hline \hline 1 & {$\left[\begin{array}{ll}\frac{\pi}{8} & \frac{3 \pi}{8}\end{array}\right]$} & {$\left[\begin{array}{ll}\frac{\pi}{8} & \frac{3 \pi}{8}\end{array}\right]$} \\
\hline 2 & {$\left[\begin{array}{ll}\frac{5 \pi}{8} & \frac{7 \pi}{8}\end{array}\right]$} & {$\left[\begin{array}{ll}\frac{5 \pi}{8} & \frac{7 \pi}{8}\end{array}\right]$} \\
\hline 3 & {$\left[\begin{array}{ll}\frac{9 \pi}{8} & \frac{11 \pi}{8}\end{array}\right]$} & {$\left[\begin{array}{ll}\frac{\pi}{8} & \frac{3 \pi}{8}\end{array}\right]$} \\
\hline 4 & {$\left[\begin{array}{ll}\frac{13 \pi}{8} & \frac{15 \pi}{8}\end{array}\right]$} & {$\left[\begin{array}{ll}\frac{5 \pi}{8} & \frac{7 \pi}{8}\end{array}\right]$} \\
\hline 5 & {$\left[\begin{array}{ll}0 & \pi\end{array}\right]$} & {$\left[\begin{array}{ll}0 & 2 \pi\end{array}\right]$} \\
\hline
\end{tabular}

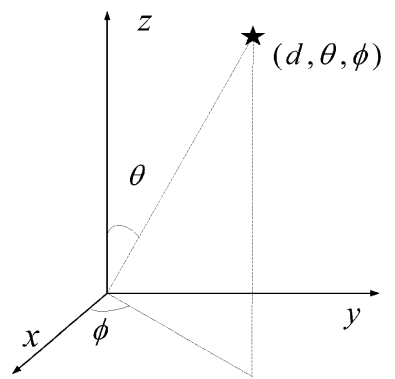

Fig. 1. Spherical coordinates of a single measurement. The receiver is at the origin and the transmitter at $(d, \theta, \phi)$.

TABLE II

SIGNAL BANDWIDTH AND RANGING ERROR STD

\begin{tabular}{|c||c|c|c|c|}
\hline Bandwidth [GHz] & 0.5 & 1.5 & 3.5 & 7.5 \\
\hline \hline$S T D[\mathrm{~cm}]$ & 11.0 & 3.5 & 1.8 & 1.5 \\
\hline
\end{tabular}

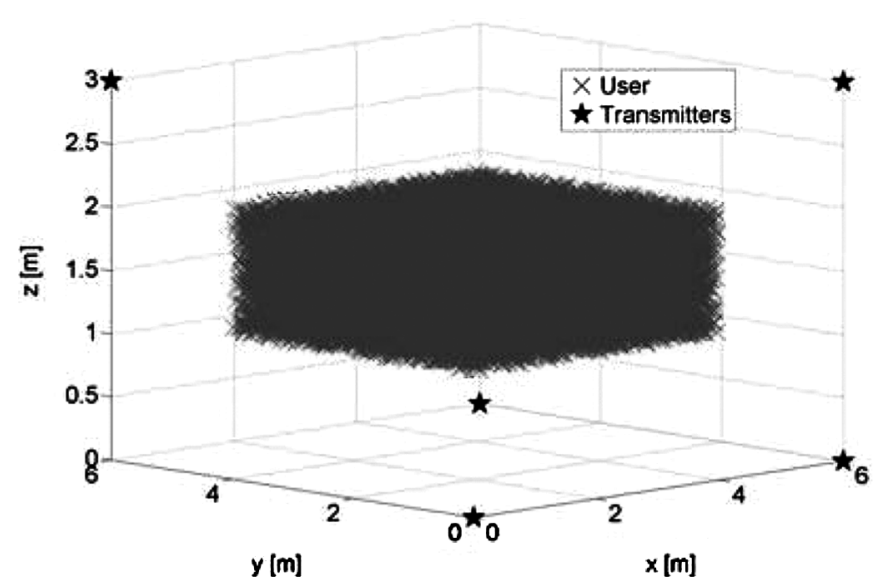

Fig. 2. Geometry setup used in simulations, with five transmitters.

in the $l$ th test. Note that $\underline{b}_{c}$ is not included in the RMSE in TDoA.

\section{B. Simulation}

For simulations, the procedure becomes:

1. Determine the system geometry as in Fig. 2, where 5 transmitters are installed in the corners of a $6 \times 6 \times 3 \mathrm{~m}^{3}$ room, and the user positions are uniformly distributed in a space of $4 \times 4 \times 1 \mathrm{~m}^{3}$, in the room center.

2. Calculate the true ranges between the 5 transmitters and the user, i.e., $d_{i}=\left\|x_{u}-x_{i}\right\|$, for $i=1, \ldots, 5$.

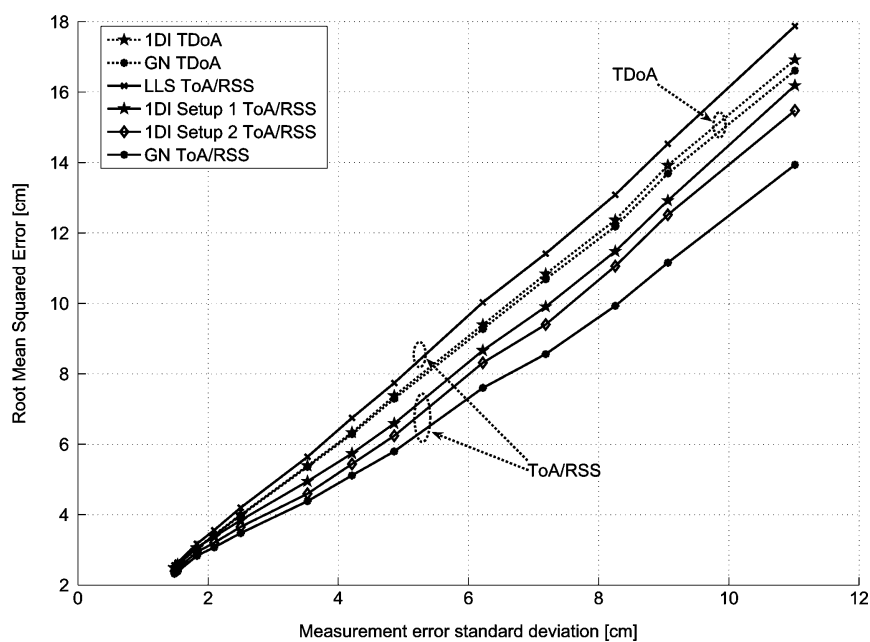

Fig. 3. RMSE of the estimates obtained using the actual UWB data, for different methods.

3. Generate range measurements by adding error, i.e., $\underline{y}_{i}=$ $d_{i}+b_{c}+\underline{e}_{i}$, with $\underline{e} \sim N_{m}\left(0, Q_{e e}(B, d)\right)$. The expression of $Q_{e e}(B, d)$ can be found in (42). In this step, $Q_{e e}(B, d)$ is calculated using true ranges, while in the next step when calculating weight matrices, $d$ is replaced by the estimated distances obtained with a initial guess in TDoA cases, and by range measurements in ToA/RSS cases.

4. Perform positioning using simulated ranges, in a similar way as described previously for the validation using actual data.

\section{RESULTS}

Results are plotted in Figs. 3 to 7. On the horizontal axis, the range error standard deviation (STD), calculated as

$$
\mathrm{STD}=\sqrt{\frac{1}{399} \sum_{l=1}^{400}\left\|\hat{d}_{l}-d_{l}\right\|^{2}}
$$

considering all 400 measurements obtained with a fixed bandwidth $B$, is given, $c f$. the values in Table II.

\section{A. RMSE}

The empirical RMSE curves of the tested methods obtained with actual data are plotted in Fig. 3. The GN estimator has the lowest RMSE. The performance of the 1DI estimator is better than the LLS estimator, and is very close to the GN estimator, especially in the TDoA case. Due to numerical problems, the RMSE of the LLS estimator in the TDoA case is of more than hundreds of meters, and is not shown in the figure.

\section{B. Setup 1 versus Setup 2}

For ToA/RSS cases, the 1DI method is tested with two setups with different parameter choices. In general, Setup 2 is always equal to, or better than Setup 1. This point is supported by the results in Fig. 3. We should note that Setup 1 is worse than Setup 2 because the system geometry in Fig. 3 is generated in a randomly uniform way. In a typical indoor room, e.g., Fig. 2, the 


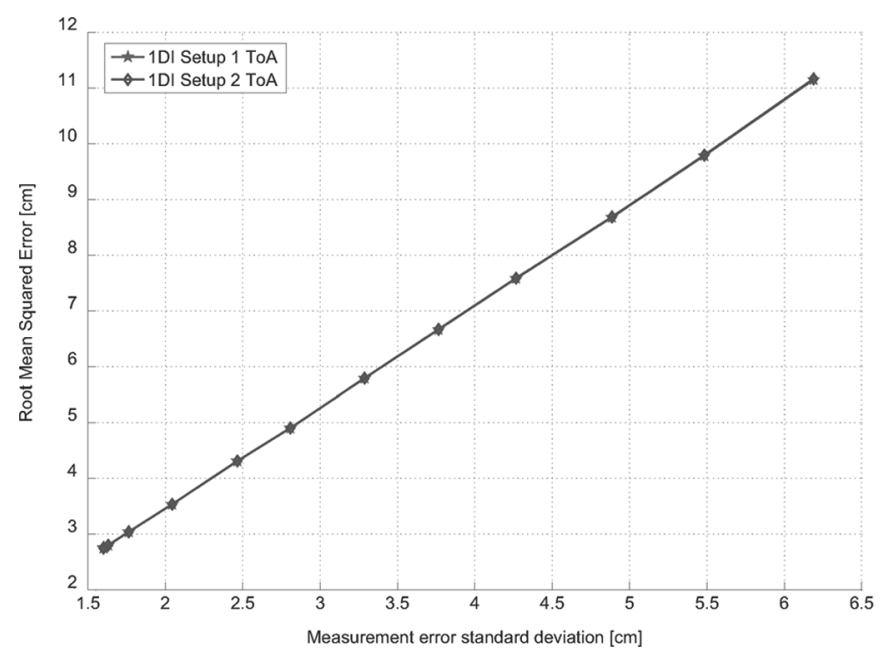

Fig. 4. Simulated RMSE curves of the estimates obtained with two setups of the 1DI method.

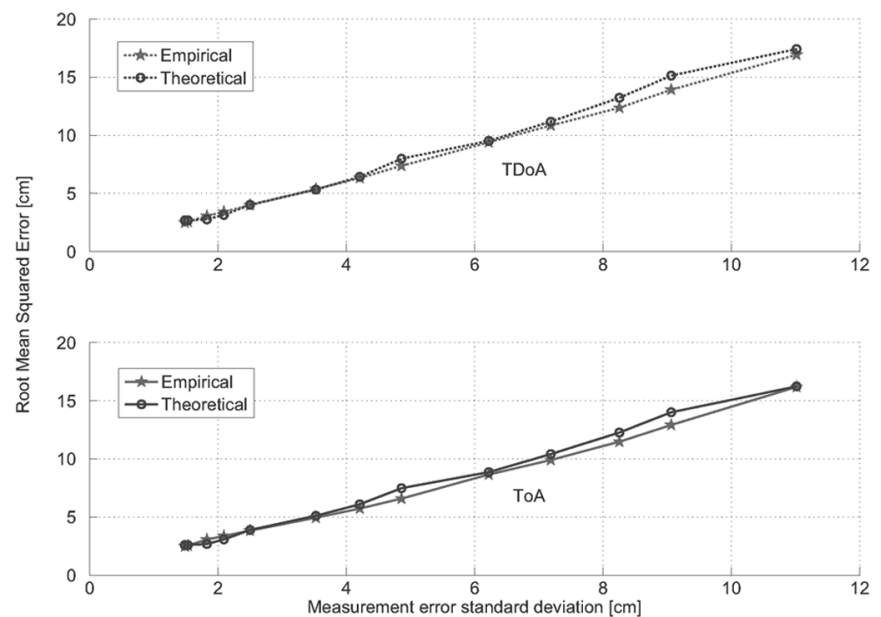

Fig. 5. The theoretical RMSE curve v.s. the empirical curve obtained using actual UWB data, for the 1DI method with Setup 1.

precision of the local height is usually the worst, and the corresponding coordinate can be directly chosen in Step 1 . This point is verified by simulation, where Setup 1 and Setup 2 behave very similarly, see Fig. 4.

\section{Empirical vs Theoretical}

In Fig. 5, the empirical RMSE curves obtained using the actual data are compared to the theoretical RMSE curves (31) and (34). The empirical and theoretical curves are close but still deviate. This may be caused by the imperfectness of the model (42), where the parameters are estimated using only 400 measurements. In Fig. 6, we show the empirical and theoretical curves obtained with the simulation, and the results show that the empirical and theoretical curves match perfectly with each other for both TDoA and ToA/RSS cases.

\section{Convergence Rate}

In general, it is difficult to claim which one of the 1DI and the GN method converges faster, since the convergence rate is dependent on both geometry and the error $\underline{e}$, see (36) and [1]. In the current test with actual data, the mean numbers of the

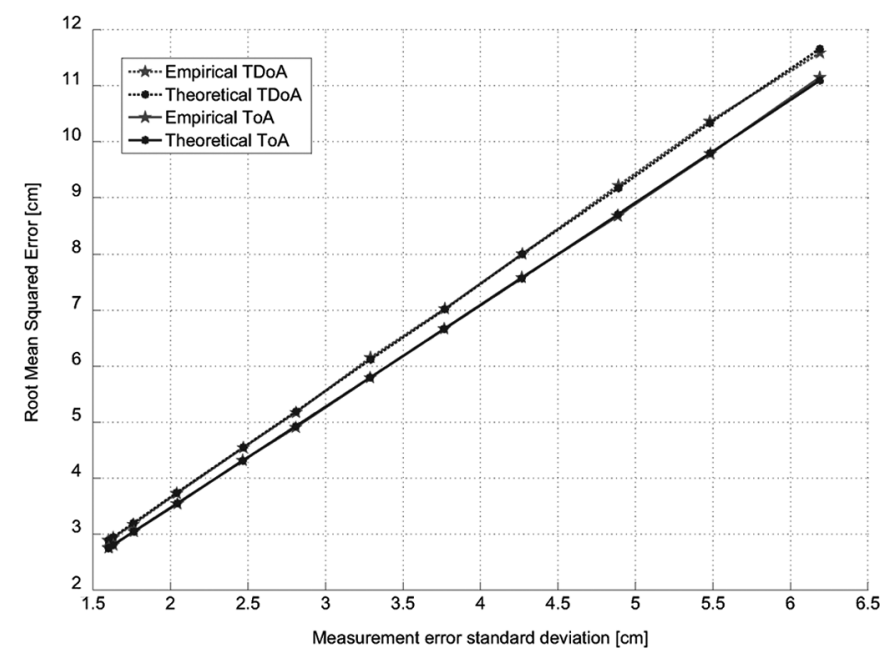

Fig. 6. The theoretical RMSE curve v.s. the empirical curve obtained using simulation, for the 1DI method with Setup 1.

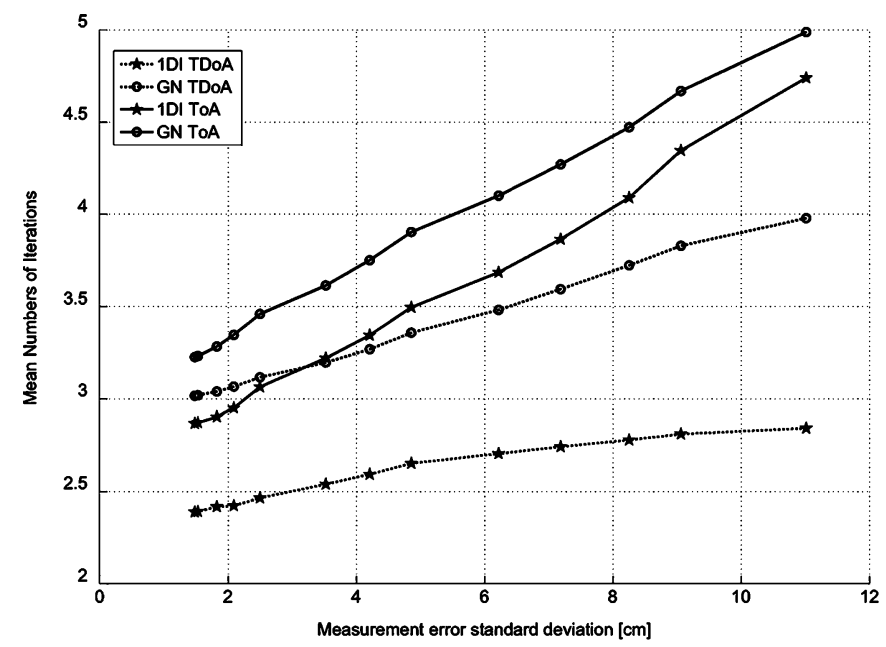

Fig. 7. Mean of the required number of iterations by the 1DI and the GN methods, obtained using the actual UWB data.

required iterations of the two methods are shown in Fig. 7, where the 1DI method requires less iterations to converge, especially in TDoA cases.

\section{E. Flop Count}

The calculations of the flop count for our test, with $m=5$ and $n=3$, can be done based on (60), (65), (67), and (69). Note that these equations are chosen since the weight matrix $W$ is diagonal, see (43). The results show that the proposed 1DI method, as compared to the GN method, saves $63 \%$ and $59 \%$ flops in TDoA and ToA/RSS cases, respectively.

\section{CONCLUSION}

In this paper, the TLS framework, consisting of 2 steps, is proposed to realize low-complexity positioning with high precision estimators, covering both TDoA and ToA/RSS cases. The low-complexity nature of the framework comes from Step 1 , where the positioning problem is transformed from $(n+1)$ - or $n$-dimension to a lower dimension. In Step 2, the high precision estimator is obtained by iteratively refining the unknowns 
with all of the original range measurements. Within the TLS framework, the 1DI method is introduced, which applies the LLS method in Step 1 to reduce the number of unknowns to one and the Gauss-Newton (GN) method to estimate the single unknown in Step 2. The way of choosing the parameter in Step 1 of the 1DI method has been discussed. The theoretical performance of the 1DI method is also analyzed, including the expression for RMSE, the convergence rate and the required number of flops in each iteration. It has been shown that in each iteration, the 1DI method can save up to $67 \%$ of the computations, compared to the GN method.

Validation of the work is done mainly based on actual UWB radio measurements, together with some supplementary simulations. Results show that the 1DI method is better than the LLS method, and its performance is close to the GN method, especially in the TDoA case. The derived theoretical RMSE perfectly matches the empirical value in simulations, and small deviations exist with actual data, which can be caused by the limited number of available data. Although the validation is done with Line-of-Sight range measurements, some techniques dealing with Non-Line-of-Sight conditions may also be applicable in this framework; for example, the compensation approach proposed in [20].

The TLS framework was introduced and applied here to the indoor self positioning scenario using UWB signals, but it also works for tracking systems in which the user sends signals to the base stations where signal processing and position estimation are accomplished.

\section{APPENDIX A}

ERROR ANALYSIS

As discussed in Section III-D

$$
\begin{aligned}
\underline{\hat{z}}= & M(y)+\partial_{y} M(y)^{T} \underline{e} \\
& +\frac{1}{2} \underline{e}^{T} \partial_{y y^{T}}^{2} M(y) \underline{e}+\cdots \\
\mu_{\hat{z}}= & \frac{1}{2} \operatorname{trace}\left\{\partial_{y y^{T}}^{2} M(y) Q_{e e}\right\} \\
Q_{\hat{z} \hat{z}}= & \partial_{y} M(y)^{T} Q_{e e} \partial_{y} M(y)
\end{aligned}
$$

where the expression of the mapping $M(\cdot)$ needs to be derived, which is related to the least-squares objective function to minimize [16].

\section{A. TDoA}

In TDoA cases, $z=b_{c}$ and the objective function to minimize is

$$
F_{1 D}\left(b_{c}\right)=\left\|\underline{y}-P\left(b_{c}, \underline{e}\right)\right\|_{W}^{2}
$$

where $P_{i}\left(b_{c}, \underline{e}\right)=\left\|E \underline{y}_{L}+E \underline{\delta}_{r} b_{c}-x_{i}\right\|+b_{c}$, and the problem is equivalent to solving

$$
\partial_{b_{c}} P\left(\underline{\hat{b}}_{c}, \underline{e}\right)^{T} W\left[\underline{y}-P\left(\underline{\hat{b}}_{c}, \underline{e}\right)\right]=0 \text {. }
$$

The terms $\partial_{y} M(y)$ and $\partial_{y y^{T}}^{2} M(y)$ can be calculated by first Taylorizing (49) with respect to $\underline{b}_{c}$ at the true value $b_{c}$

$$
\begin{aligned}
\partial_{b_{c}} P & \left(\underline{\hat{b}}_{c}, \underline{e}\right)^{T} W\left[\underline{y}-P\left(\underline{\hat{b}}_{c}, \underline{e}\right)\right] \\
= & \partial_{b_{c}} P\left(b_{c}, \underline{e}\right)^{T} W\left[\underline{y}-P\left(b_{c}, \underline{e}\right)\right] \\
& +\left[\partial_{b_{c}^{2}}^{2} P\left(b_{c}, \underline{e}\right)^{T} W\left[\underline{y}-P\left(b_{c}, \underline{e}\right)\right]\right. \\
& \left.-\partial_{b_{c}} P\left(b_{c}, \underline{e}\right)^{T} W \partial_{b_{c}} P\left(b_{c}, \underline{e}\right)\right]\left(\underline{\hat{b}}_{c}-b_{c}\right) \\
& +\frac{1}{2}\left\{\partial_{b_{c}^{3}}^{3} P\left(b_{c}, \underline{e}\right)^{T} W\left[\underline{y}-P\left(b_{c}, \underline{e}\right)\right]\right. \\
& -\partial_{b_{c}^{2}}^{2} P\left(b_{c}, \underline{e}\right)^{T} W \partial_{b_{c}} P\left(b_{c}, \underline{e}\right) \\
& -\partial_{b_{c}^{2}}^{2} P\left(b_{c}, \underline{e}\right)^{T} W \partial_{b_{c}} P\left(b_{c}, \underline{e}\right) \\
& \left.-\partial_{b_{c}} P\left(b_{c}, \underline{e}\right)^{T} W \partial_{b_{c}^{2}}^{2} P\left(b_{c}, \underline{e}\right)\right\}\left(\underline{b}_{c}-b_{c}\right)^{2} \\
& +\cdots=0 .
\end{aligned}
$$

Then Taylorize $P\left(b_{c}, \underline{e}\right)$ with respect to $\underline{e}$ at $E\{\underline{e}\}=0$, which gives

$$
\begin{aligned}
P_{i}\left(b_{c}, \underline{e}\right)= & P_{i}\left(b_{c}, 0\right)+\partial_{e} P_{i}\left(b_{c}, 0\right)^{T} \underline{e} \\
& +\frac{1}{2} \underline{e}^{T} \partial_{e e}^{2} P_{i}\left(b_{c}, 0\right) \underline{e}+\cdots \\
= & A_{i}\left(b_{c}\right)+R_{i}\left(b_{c}\right) \underline{e}+\frac{1}{2} \underline{e}^{T} S_{i}\left(b_{c}\right) \underline{e}+\cdots
\end{aligned}
$$

where we denote

$$
\begin{aligned}
& {\left[R_{i}\right]_{1 \times m}} \\
& =\partial_{e} P_{i}\left(b_{c}, 0\right)^{T} \\
& =\frac{\left.\left(E y_{L}+E \delta y_{r} b_{c}-x_{i}\right)^{T}\left(E \partial_{e^{T}} \underline{y}_{L}+E b_{c} \partial_{e^{T}} \underline{\delta y}_{r}\right)\right|_{\underline{e}=0}}{\left\|E y_{L}+E \delta y_{r} b_{c}-x_{i}\right\|} \\
& {\left[\partial_{e^{T}} \underline{y} L+b_{c} \partial_{e^{T}} \underline{\delta}{ }_{r}\right]_{(m-1) \times m}} \\
& =\left[\begin{array}{cccc}
2 d_{1}+2 \underline{e}_{1} & & 0 & -2 d_{r}-2 \underline{e}_{r} \\
& \ddots & & \vdots \\
0 & & 2 d_{m-1}+2 \underline{e}_{m-1} & -2 d_{r}-2 \underline{e}_{r}
\end{array}\right] \\
& {\left[S_{i}\right]_{m \times m}=\partial_{e e}^{2} P_{i}\left(b_{c}, 0\right)} \\
& \begin{aligned}
= & \frac{\left.\left(E \partial_{e^{T}} \underline{y}_{L}+E b_{c} \partial_{e^{T}} \underline{\delta y}_{r}\right)^{T}\left(E \partial_{e^{T}} \underline{y}_{L}+E b_{c} \partial_{e^{T}} \underline{\delta y}_{r}\right)\right|_{\underline{e}=0}}{\left\|E y_{L}+E \delta y_{r} b_{c}-x_{i}\right\|} \\
& +\frac{H}{\left\|E y_{L}+E \delta y_{r} b_{c}-x_{i}\right\|}
\end{aligned} \\
& -\frac{\left.\left(E \partial_{e^{T}} \underline{y}_{L}+E b_{c} \partial_{e^{T}} \underline{\delta y_{y}}\right)^{T}\left(E y_{L}+E \delta y_{r} b_{c}-x_{i}\right)\right|_{\underline{e}=0}}{\left\|E y_{L}+E \delta y_{r} b_{c}-x_{i}\right\|^{3}} \\
& \left.\cdot\left(E y_{L}+E \delta y_{r} b_{c}-x_{i}\right)^{T}\left(E \partial_{e^{T}} \underline{y}_{L}+E b_{c} \partial_{e^{T}} \underline{\delta}_{r}\right)\right|_{\underline{e}=0} \\
& H_{m \times m} \\
& =\left.\left(E y_{L}+E b_{c} \delta y_{r}-x_{i}\right)^{T} E \partial_{e e}^{2}\left(\underline{y}_{L}+b_{c} \delta \underline{y}_{r}\right)\right|_{\underline{e}=0} \\
& =2 \operatorname{diag}\left\{\left[\begin{array}{c}
E^{T}\left(E y_{L}+E b_{c} \delta y_{r}-x_{i}\right) \\
-\sum_{j=1}^{n-1}\left[\left(E y_{L}+E b_{c} \delta y_{r}-x_{i}\right)^{T} E\right]_{j}
\end{array}\right]_{m \times 1}\right\}
\end{aligned}
$$

for simplicity. 
Since (50) is equal to zero for all $\underline{e}$, we can collect terms with the same order and set them to zero. Plugging (45) and (52) into (50) and collect all the first-order terms gives

$$
\begin{aligned}
& \partial_{y} M(y)^{T} \\
& \quad=\left(\partial_{b_{c}} A\left(b_{c}\right)^{T} W \partial_{b_{c}} A\left(b_{c}\right)\right)^{-1} \partial_{b_{c}} A\left(b_{c}\right)^{T} W(I-R)
\end{aligned}
$$

and collecting the second-order terms gives us

$$
\begin{aligned}
\partial_{y y^{T}}^{2} M(y)= & \left(\partial_{b_{c}} A\left(b_{c}\right)^{T} W \partial_{b_{c}} A\left(b_{c}\right)\right)^{-1} \\
& \cdot\left\{-\sum_{i=1}^{m}\left\{\left[\partial_{b_{c}} A\left(b_{c}\right)^{T} W\right]_{i} \partial_{b_{c}} S_{i}\right\}\right. \\
& +2 \partial_{b_{c}} R^{T} W(I-R) \\
& +2(I-R)^{T} W \partial_{b_{c}^{2}}^{2} A\left(b_{c}\right) \partial_{y} M(y)^{T} \\
& -4 R^{T} W \partial_{b_{c}} A\left(b_{c}\right) \partial_{y} M(y)^{T} \\
& \left.-\partial_{y} M(y)\left[3 \partial_{b_{c}} A\left(b_{c}\right)^{T} W \partial_{b_{c}^{2}}^{2} A\left(b_{c}\right)\right] \partial_{y} M(y)^{T}\right\} .
\end{aligned}
$$

Furthermore

$$
\begin{aligned}
& K=\mathrm{E}\left\{\underline{\delta y_{r}} \underline{e}^{T}\right\} \\
& =\left[\begin{array}{cccc}
-2 \sigma_{1}^{2} & & 0 & 2 \sigma_{r}^{2} \\
& \ddots & & \vdots \\
0 & & -2 \sigma_{m-1}^{2} & 2 \sigma_{r}^{2}
\end{array}\right]_{(m-1) \times m} \\
& J=\mathrm{E}\left\{G \underline{e}_{L} \underline{\mu}_{\hat{b}_{c}}^{T}\left(G \delta y_{r}+F\right)^{T}\right\} \\
& =G\left[\begin{array}{cccc}
2 d_{1} \sigma_{1}^{2} & & 0 & -2 d_{r} \sigma_{r}^{2} \\
& \ddots & & \vdots \\
0 & & 2 d_{m-1} \sigma_{m-1}^{2} & -2 d_{r} \sigma_{r}^{2}
\end{array}\right] \\
& \text { - } \partial_{y} M(y)\left(G \delta y_{r}+F\right)^{T} \\
& i, j=1, \ldots, m-1, \quad i \neq j .
\end{aligned}
$$$$
\left[\mathrm{E}\left\{\underline{e}_{L} \underline{e}_{L}^{T}\right\}\right]_{i i}=4 d_{i}^{2} \sigma_{i}^{2}+4 d_{r}^{2} \sigma_{r}^{2}
$$$$
\left[\mathrm{E}\left\{\underline{e}_{L} \underline{e}_{L}^{T}\right\}\right]_{i j}=4 d_{r}^{2} \sigma_{r}^{2} \text {, }
$$

Note that the third and higher order terms of $\underline{e}$ are ignored.

\section{B. $T o A / R S S$}

The derivation of $\partial_{y} M(y), \partial_{y y^{T}}^{2} M(y)$ and $J$ in ToA/RSS cases can be done based on the same idea. The obtained results are very similar as for TDoA, and one can directly use the expression of $\partial_{y} M(y)(53), \partial_{y y^{T}}^{2} M(y)(54)$, and $J$ (55), and only needs to change $G \rightarrow E, \delta y_{r} \rightarrow C$ and $K \rightarrow 0$. Again, we should note that the expressions for $E$ and $F$ in ToA/RSS cases are very similar but not identical to the ones in TDoA cases.

\section{APPENDIX B \\ FLOP COUNT}

The flop counts for the 1DI method and the GN method are determined in this appendix.

\section{A. TDoA}

In each iteration, the common terms to update for both methods are $d^{0}$ and $A\left(x^{0}\right)=P\left(b_{c}^{0}, e\right)$. To update a single element $d_{i}^{0}$

$$
d_{i}^{0}=\underbrace{\underbrace{\left(x_{u}^{0}-x_{i}\right)^{T} \underbrace{\left(x_{u}^{0}-x_{i}\right)}_{n \text { flops }}}_{n+2 n-1 \text { flops }}}_{\text {Total } n+2 n-1+1 \text { flops }}
$$

$3 n$ flops are required. Therefore, for $m$-D vector $d^{0}, 3 m n$ flops are required.

The term $A\left(x^{0}\right)=P\left(b_{c}^{0}, e\right)$ can be updated based on $d^{0}$ and $b_{c}^{0}$, which requires $m$ flops.

1DI: The calculations in each iteration of the 1DI method include updating the vector $\partial_{b_{c}} P\left(b_{c}^{0}, e\right)$ and (19). For a single element $\partial_{b_{c}} P_{i}\left(b_{c}^{0}, e\right)$

$$
\partial_{b_{c}} P_{i}\left(b_{c}^{0}, e\right)=\underbrace{\overbrace{d_{i}^{0}}^{\frac{\left(E y_{L}-x_{i}\right)^{T} E \delta y_{r}+\overbrace{\left(E \delta y_{r}\right)^{T} E \delta y_{r} b_{c}^{0}}^{1 \text { flop }}}{d_{i}^{0} \text { flops }}}+1}_{\text {Total } 1+1+1+1 \text { flops }}
$$

only 4 flops are required. Note that the calculations for the constant terms $\left(E y_{L}-x_{i}\right)^{T} E \delta y_{r}$ and $\left(E \delta y_{r}\right)^{T} E \delta y_{r}$ have been done in the first iteration and do not need to be repeated again. Therefore, $4 m$ flops are needed to update $\partial_{b_{c}} P\left(b_{c}^{0}, e\right)$.

Once $P\left(b_{c}^{0}, e\right)$ and $\partial_{b_{c}} P\left(b_{c}^{0}, e\right)$ are updated, the flops required in (19) include, $(2 m-1) m$ for $\partial_{b_{c}} P^{T}\left(b_{c}^{0}, e\right) \cdot W, m$ for $y-$ $P\left(b_{c}^{0}, e\right), 2 m-1$ for $\partial_{b_{c}} P^{T}\left(b_{c}^{0}, e\right) W \cdot\left(y-d^{0}\right)$, and $2 m-1$ for $\partial_{b_{c}} P^{T}\left(b_{c}^{0}, e\right) W \cdot \partial_{b_{c}} P\left(b_{c}^{0}, e\right)$. Then 1 flop is used to calculate $\left(\partial_{b_{c}} P^{T}\left(b_{c}^{0}, e\right) W \partial_{b_{c}} P\left(b_{c}^{0}, e\right)\right)^{-1}, 1$ flop to multiply it with $\partial_{b_{c}} P^{T}\left(b_{c}^{0}, e\right) W\left(y-d^{0}\right)$, and 1 flop to add $b_{c}^{0}$. The stopping criteria (20) requires the evaluation of $\left\|\hat{b}_{c}-b_{c}^{0}\right\|^{2}$, and since $\hat{b}_{c}-b_{c}^{0}$ has already been evaluated in previous steps, only 1 flop is required. Finally, the update of $\underline{\hat{x}}_{u}=E \underline{y}_{L}+E \delta y_{r} \hat{b}_{c}$, for the evaluation of $d^{0}$, requires $2 \mathrm{n}$ flops.

Therefore, in each iteration, the number of required flops for the 1DI method, including the common terms, read

$$
\text { flops }_{1 \mathrm{DI}}=2 m^{2}+3 m n+9 m+2 n+2 .
$$

If $W$ is simply a diagonal matrix with unequal diagonal entries, only $m$ flops are required to evaluate $\partial_{b_{c}} P^{T}\left(b_{c}^{0}, e\right) \cdot W$ rather than $(2 m-1) m$, and the total number of flops becomes

$$
\text { flops }_{1 \mathrm{DI}}^{\prime}=3 m n+11 m+2 n+2 .
$$

$G N$ : In each iteration, the calculations for the GN method include updating part of the $m \times(n+1)$ matrix $A$ and the equation

$$
\left(A^{T} W A\right)\left(\hat{x}_{u}-x_{u}^{0}\right)=A^{T} W\left(y-A\left(x^{0}\right)\right) .
$$


The expression for a single element $A_{i j}, i=1, \ldots, m$ and $j=$ $1, \ldots, n$ is

$$
A_{i j}=\frac{x_{u j}-x_{i j}}{d_{i}^{0}}
$$

which requires 2 flops. Note that the last column of $A$ contains only constants. For the whole matrix $A, 2 m n$ flops are needed.

To obtain $\hat{x}$ from (61), the explicit computation of the inversion of $A^{T} W A$ is usually avoided by first applying a triangular decomposition: $A^{T} W A=L L^{T}$, e.g., Cholesky decomposition, which requires $n^{3} / 3$ flops [21]. Denoting $r=$ $A^{T} W\left(y-A\left(x^{0}\right)\right)$, one has

$$
L \hat{z}=r \quad \text { and } \quad L^{T} \hat{x}=\hat{z} .
$$

Since $L$ is lower triangular, the first entry of $\hat{z}$ can be computed as $\hat{z}_{1}=r_{1} / L_{11}$. This result can be used to compute the second entry as $\hat{z}_{2}=\left(r_{2}-L_{21} \hat{z}_{1}\right) / L_{22}$. In this way one continues to obtain $\hat{z}$. For a $n+1$-D vector $\hat{z}$, the required flops is $(n+1)^{2}$. To obtain $\hat{x}$ from $\hat{z}$, the same number of flops is required.

Hence, to calculate $\hat{x}_{u}$ one needs, $n(2 m-1) m$ for $A^{T} \cdot W$, $m$ for $y-A\left(x^{0}\right),(n+1)(2 m-1)$ for $A^{T} W \cdot\left(y-A\left(x^{0}\right)\right)$, $n(2 m-1)(n+1)$ for $A^{T} W \cdot A,(n+1)^{3} / 3$ for Cholesky decomposition, $2(n+1)^{2}$ for $\hat{x}-x^{0}$ and $n+1$ for $\hat{x}_{u}$. The stopping criteria requires the evaluation of $\left\|\hat{x}-x^{0}\right\|^{2}$ and since $\hat{x}-x^{0}$ has already been calculated in previous steps, the number of required flops is $2 n+1$.

The total number of flops, including the common term, read

$$
\begin{aligned}
\text { flops }_{G N}=n^{3} / 3+2 m^{2} n+2 m n^{2} \\
+2 n^{2}+7 m n+4 m+6 n+4 / 3 .
\end{aligned}
$$

If $W$ is simply a diagonal matrix, with unequal diagonal entries, only $n m$ flops are required to evaluate $A^{T} \cdot W$ rather than $n(2 m-1) m$, and the total number of flops becomes

$$
\begin{array}{rl}
\text { flops }_{G N}^{\prime}=n^{3} / 3+2 & m n^{2} \\
& +2 n^{2}+9 m n+4 m+6 n+4 / 3 .
\end{array}
$$

\section{B. ToA/RSS}

In each iteration, the common term to update is the $m$-D vector $A\left(x^{0}\right)=P\left(b_{c}^{0}, e\right)=d^{0}$, and $3 m n$ flops are required.

IDI: Compared to the TDoA cases, the difference is only the vector $\partial_{b_{c}} P\left(b_{c}^{0}, e\right)$, which now requires only $3 m$ flops.

Therefore, in each iteration, the number of required flops for the 1DI method, including the common term, read

$$
\text { flops }_{1 \mathrm{DI}}=2 m^{2}+3 m n+7 m+2 n+2
$$

If $W$ is diagonal the total number of flops becomes

$$
\text { flops }_{1 \mathrm{DI}}^{\prime}=3 m n+9 m+2 n+2 .
$$

$G N$ : To update $A, 2 m n$ flops are needed, the same as for TDoA cases. To calculate $\hat{x}_{u}, n(2 m-1) m$ is needed for $A^{T} \cdot W$, $m$ for $y-d^{0}, n(2 m-1)$ for $A^{T} W \cdot\left(y-d^{0}\right), n(2 m-1) n$ for $A^{T} W \cdot A,(n)^{3} / 3$ for Cholesky decomposition, $2 n^{2}$ for $\hat{x}-x^{0}$, and $n$ for $\hat{x}$. The stopping criteria requires $2 n-1$.
The total number of flops, including the common term, read

$$
\begin{aligned}
\text { flops }_{G N}=n^{3} / 3 & +2 m^{2} n \\
& +2 m n^{2}+n^{2}+6 m n+m+2 n-1 .
\end{aligned}
$$

If $W$ is diagonal the total number of flops becomes

$$
\text { flops }_{G N}^{\prime}=n^{3} / 3+2 m n^{2}+n^{2}+8 m n+m+2 n-1 .
$$

\section{REFERENCES}

[1] P. J. G. Teunissen, "Nonlinear least-squares," Manuscripta Geodaetica, vol. 15 , no. 3, pp. 137-150, 1990.

[2] S. Bancroft, "An algebraic solution for the GPS equations," IEEE Trans. Aerosp. Electron. Syst., vol. 21, no. 7, pp. 56-59, 1985.

[3] H. C. Schau and A. Z. Robinson, "Passive source localization employing intersecting spherical surfaces from time-of-arrival differences," IEEE Trans. Acoust., Speech, Signal Process., vol. ASSP-35, pp. 1223-1225, Aug. 1987.

[4] J. L. Leva, "An alternative closed-form solution to the GPS pseudorange equations," IEEE Trans. Aerosp. Electron. Syst., vol. 32, no. 4, pp. 1430-1439, 1996.

[5] J. Beutel, "Geolocation in a Picoradio Environment," Master's thesis, Univ. Calif., Berkeley, 1999.

[6] J. O. Smith and J. S. Abel, "The spherical interpolation method for closed-form passive source localization using range difference measurements," in Proc. IEEE Int. Conf. Acoust., Speech, Signal Process., Apr. 1987, vol. 12, pp. 471-474.

[7] J. J. Caffery, "A new approach to the geometry of TOA location," in Proc. IEEE Veh. Technol. Conf. (VTC), Boston, MA, Sep. 2000, vol. 4, pp. 1943-1949.

[8] Z. Li, W. Trappe, Y. Zhang, and B. Nath, "Robust statistical methods for securing wireless localization in sensor networks," in Proc. IEEE Int. Symp. Inf. Process. in Sens. Netw. (IPSN), Los Angeles, CA, Apr. 15, 2005, pp. 91-98.

[9] S. Venkatesh and R. M. Buehrer, "A linear programming approach to NLOS error mitigation in sensor networks," in Proc. IEEE Int. Symp. Inf. Process. in Sens. Netw. (IPSN), Nashville, TN, Apr. 2006, pp. 301-308.

[10] I. Guvenc, S. Gezici, F. Watanabe, and H. Inamura, "Enhancements to linear least squares localization through reference selection and ML estimation," in Proc. IEEE Int. Conf. Wireless Commun. Netw. (WCNC), Las Vegas, NV, Mar. 31/Apr. 3 2008, pp. 284-289.

[11] M. Rydström, A. Urruela, E. G. Ström, and A. Svensson, "A low complexity algorithm for sensor localization," in Proc. Eur. Wireless Conf., 2005, pp. 714-718.

[12] Y. Huang and J. Benesty, "Real-time passive source localization: A practical linear-correction least-squares approach," IEEE Trans. Speech Audio Process., vol. 9, no. 8, pp. 943-955, Nov. 2001.

[13] K. W. Cheung, H. C. So, W.-K. Ma, and Y. T. Chan, "Least squares algorithms for time-of-arrival-based mobile location," IEEE Trans. Signal Process., vol. 52, no. 4, pp. 1121-1128, Apr. 2004.

[14] A. O. Hero, III and D. Blatt, "Sensor network source localization via projection onto convex sets (POCS)," in Proc. IEEE Int. Conf. Acoust., Speech, Signal Process., Mar. 2005, vol. 3, pp. 689-692.

[15] P. Misra and P. Enge, Global Positioning System: Signals, Measurements, and Performances, 2nd ed. Lincoln, MA: Ganga-Jamuna, 2006.

[16] P. J. G. Teunissen, "First and second moments of nonlinear least-squares estimators," Bulletin Geodesique, vol. 63, no. 3, pp. 253-262, 1989.

[17] FCC. (2002) Revision of Part 15 of the Commission's Rules Regarding Ultra-Wideband Transmission Systems [Online]. Available: http://www.fcc.gov/oet/dockets/et98-153/ [Online]. Available:

[18] G. Bellusci, G. J. M. Janssen, J. Yan, and C. C. J. M. Tiberius, "Model of the distance and bandwidth dependency of TOA-based UWB ranging error," in Proc. IEEE Int. Conf. on Ultra-Wideband (ICUWB), Hannover, Germany, Sep. 10-12, 2008.

[19] B. Alavi and K. Pahlavan, "Modeling of the distance error for indoor geolocation," in Proc. IEEE Int. Conf. Wireless Commun. Netw. (WCNC), Mar. 2003, pp. 668-672. 
[20] D. Dardari, A. Conti, J. Lien, and M. Z. Win, "The effect of cooperation on localization systems using UWB experimental data," J. Adv. Signal Process., Special Issue on Cooper. Localiz. Wireless Ad Hoc and Sens. Netw., vol. 2008, 2008.

[21] G. H. Golub and C. F. van Loan, Matrix Computations. Baltimore, MD: Johns Hopkins Univ. Press, 1989.

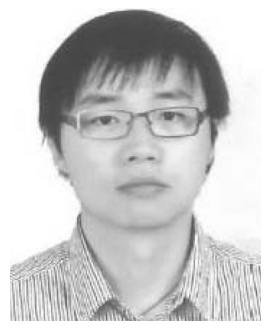

Junlin Yan received the B.E. degree in electrical engineering from Fudan University, Shanghai, China, in 2003. He received the M.Sc. degree in digital communications from Chalmers University of Technology, Sweden, in 2006.

$\mathrm{He}$ is currently completing the Ph.D. degree with the group of Mathematical Geodesy and Positioning, Faculty of Aerospace Engineering, Delft University of Technology, Delft, The Netherlands. His research topic is indoor positioning with UWB radio signals.

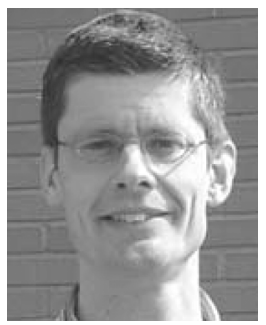

Christian C. J. M. Tiberius received the Ph.D. degree in 1998 from the Delft University of Technology (TUDelft), Delft, The Netherlands, on recursive data processing for kinematic GPS surveying.

$\mathrm{He}$ is currently an Associate Professor with the Delft Institute of Earth Observation and Space Systems (DEOS), Delft University of Technology. His research interest lies in navigation, primarily with Global Navigation Satellite Systems and indoor radio positioning. $\mathrm{He}$ is, and has been responsible for, for more than 10 years, many projects in the area of navigation with national and international agencies, like ESA, and industry, as well.

Dr. Tiberius is currently a member of the American Institute of Navigation (ION) Satellite Division Executive Committee.

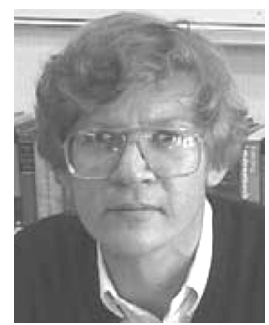

Peter J. G. Teunissen received the Ph.D. degree in geodesy (summa cum laude) in 1985, from the Delft University of Technology (TUDelft), Delft, The Netherlands.

Immediately following, he was awarded the prestigious five-year Constantijn en Christiaan Huygens Fellowship (1986-1991) by the Netherlands Organization for the Advancement of Pure Research. In 1988, he attained full Professor of Geodesy and Navigation, TUDelft, where he has held various senior academic positions: Head of Mathematical and Physical Geodesy Department, Faculty of Geodesy (1993-1998); Vice-Dean of Faculty of Civil Engineering and Geosciences (2001-2002); Director of Education (2002-2004), Program Director Delft Research Centre Earth and Atmosphere (2004-2008), Chair of the Netherlands Geodetic Commission (1993-2009), and Head of Earth Observation and Space Systems Department, Faculty of Aerospace Engineering (2003-2006). He is inventor of the LAMBDA method and has 25 years of research experience in GNSS positioning and navigation. He is currently Federation Fellow of the Australian Research Council (2009) working on the theory and modeling for the next generation GNSS

Prof. Teunissen was elected Fellow of the Royal Netherlands Academy of Sciences in 2000 and Fellow of the International Association of Geodesy (IAG) in 1991.

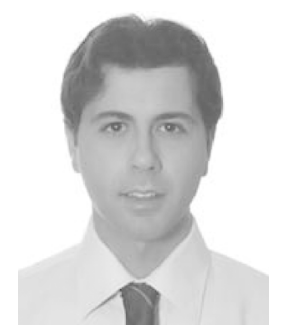

Giovanni Bellusci received the B.Sc. and M.Sc. degrees in telecommunication engineering (both summa cum laude) from the University of Pisa, Italy, in 2003 and 2005, respectively.

Since 2006, he has been pursuing the Ph.D. degree with the Wireless and Mobile Communications Group, Delft University of Technology, The Netherlands. His research interests include UWB technology for ranging and positioning applications.

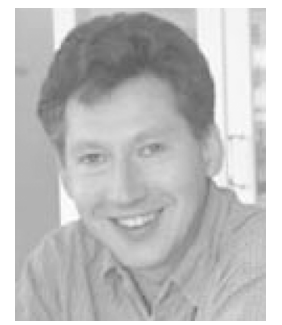

Gerard J. M. Janssen received the M.Sc.E.E. degree from Eindhoven University of Technology, The Netherlands, in 1986, and the Ph.D. degree from Delft University of Technology (TUDelft), Delft, The Netherlands, in 1998.

In 1986, he joined the Physics and Electronics Laboratory, Dutch Organization of Applied Scientific Research (TNO), where he was involved in radar-cross-section modeling, radio direction finding, interference cancelation, and wideband propagation measurements. He is currently an Associate Professor with the Wireless and Mobile Communications group, TUDelft. His research interests are in wireless communication, especially narrowband multiuser detection, digital modulation techniques, channel modeling, diversity techniques, and ultra wideband communications and positioning. 